\title{
RADII OF IMMERSED MANIFOLDS AND NONEXISTENCE OF IMMERSIONS
}

\author{
TÔRU ISHIHARA
}

\begin{abstract}
Let $M$ be a compact Riemannian manifold isometrically immersed in a complete Riemannian manifold $N$. By the radius of $M$ in $N$, we mean the minimum of radii of closed geodesic balls in $N$ which contain $M$. Using the concept of a radius, we will give a theorem about the nonexistence of isometric immersions, which is a generalization of J. D. Moore's result.
\end{abstract}

1. Introduction. Let $M$ and $N$ denote $C^{\infty}$ Riemannian manifolds, $K$ and $K^{*}$ their respective sectional curvature functions. J. D. Moore [3] proved that when $N$ is a complete simply connected Riemannian manifold with $a<K^{*}<b<0$, and $M$ is a compact Riemannian manifold with $K \leqslant a-b, M$ possesses no isometric immersion in $N$, unless $\operatorname{dim} N>2 \operatorname{dim} M$. On the other hand H. Jacobowitz [1] showed that an isometric immersion of an $n$-dimensional compact Riemannian manifold with sectional curvature always less than $\lambda^{-2}$ into Euclidean space of dimension $2 n-1$ can never be contained in a ball of radius $\lambda$. In this note, using methods similar to those of [3], we generalize the results of J. D. Moore and $\mathbf{H}$. Jacobowitz. At first we define a positive continuous function $C(b, d)$ on $(-\infty, 0]$ $\times(0, \infty)$ by

$$
C(b, d)= \begin{cases}\frac{1}{d} & \text { if } b=0, \\ \sqrt{-b} \operatorname{coth}(d \sqrt{-b}) & \text { if } b<0 .\end{cases}
$$

This function is monotonically decreasing with respect to $b$ and also $d$. We will prove

THEOREM 1. Let $N$ be a complete simply connected Riemannian manifold whose sectional curvatures $K^{*}(\sigma)$ satisfy the inequalities

$$
a \leqslant K^{*}(\sigma) \leqslant b \leqslant 0,
$$

$M$ be a compact Riemannian manifold with diameter $d$. Assume that at every point of $M$, there is a p-dimensional subspace in the tangent space, along whose plane elements $\sigma$, it holds

$$
K(\sigma) \leqslant a+C^{2}(b, d) .
$$

If $\operatorname{dim} N<\operatorname{dim} M+p$, then $M$ cannot be isometrically immersed in $N$.

As the function $C(b, d)$ satisfies $C^{2}(b, d)>-b$, this theorem strengthens the

Received by the editors August 6, 1978 and, in revised form, September 27, 1978.

AMS (MOS) subject classifications (1970). Primary 53C40.

Key words and phrases. Nonexistence of isometric immersions, radius, diameter, the second variation. 
result in [3]. Theorem 1 is immediate from the following theorem which generalizes H. Jacobowitz's result [1].

Theorem 2. Assume that $N$ satisfies the same conditions as in Theorem 1 . Let $d$ be some positive constant. Let $M$ be a compact manifold such that at every point of $M$, there is a p-dimensional subspace in the tangent space, along whose plane elements $\sigma$, the inequality (2) holds. If $\operatorname{dim} N<\operatorname{dim} M+p$, then no isometric immersion of $M$ into $N$ is contained in a ball of radius $d$.

2. Radii of immersed manifolds. We will deal with a ball containing an immersed manifold as in [1]. Let $M$ be a compact Riemannian manifold isometrically immersed in a complete Riemannian manifold $N$. Let $d($, ) be the distance function of $N$. For any point $x \in N$ and any $r>0$, put $B(x, r)=\{y \in N, d(x, y)<r\}$. Then we set

$$
\begin{aligned}
r(M) & =\inf \{r ; M \subset B(x, r)\} \\
& =\inf \{\max \{d(x, y), y \in M\}, x \in N\} .
\end{aligned}
$$

As $M$ is compact, we can prove there is a point $x_{0} \in N$ such that $B\left(x_{0}, r(M)\right) \supset$ $M$. Moreover there is a point $y_{0} \in M$ such that $d\left(x_{0}, y_{0}\right)=r(M)$. We will call $r(M)$ the radius of $M$ in $N$ and $B\left(x_{0}, r(M)\right)$ a minimum ball containing $M$. Generally, there are several minimal balls containing $M$. For example, let $S^{1}=\left\{\left(x_{1}, x_{2}\right)\right.$; $\left.x_{1}^{2}+x_{2}^{2}=1\right\}$ be naturally imbedded in $S^{2}=\left\{\left(x_{1}, x_{2}, x_{3}\right) ; x_{1}^{2}+x_{2}^{2}+x_{3}^{2}=1\right\}$. Then minimal balls containing $S^{1}$ are $B((0,0,1), \pi / 2)$ and $B((0,0,-1), \pi / 2)$. But there is only one minimal ball for a compact manifold immersed in a euclidean space. In fact we have

THEOREM 3. Let $M$ be a compact manifold immersed in an $n$-dimensional euclidean space $E^{n}$. Then there is only one point $x_{0} \in E^{n}$ such that $r(M)=\max \left\{d\left(x_{0}, y\right)\right.$, $y \in M\}$.

Proof. Take a point $x_{0}$ which satisfies the above equality. Let $S\left(x_{0}, r(M)\right)=\{y$ $\left.\in M, d\left(x_{0}, y\right)=r(M)\right\}$. At first we will prove that $S\left(x_{0}, r(M)\right)$ contains more than one point. Suppose there is only one point $y_{0}$ in $M$ with $d\left(x_{0}, y_{0}\right)=r(M)$. Take a positive $\delta$ satisfying $\delta<r(M) / 2$. Put $r_{1}=\max \left\{d\left(x_{0}, y\right), M-B\left(y_{0}, \delta\right) \ni y\right\}$. Then $r_{1}<r(M)$. Hence if $x_{1} \in B\left(x_{0}, \varepsilon_{1} / 2\right)$,

$$
\max \left\{d\left(x_{1}, y\right), M-B\left(y_{0}, \delta\right) \ni y\right\}<r_{1}+\frac{\varepsilon_{1}}{2}<r(M),
$$

where we put $\varepsilon_{1}=r(M)-r_{1}$. Let $x_{\varepsilon}=\varepsilon x_{0}+(1-\varepsilon) y_{0}$. An easy calculation shows that for any $y \in M \cap B\left(y_{0}, \delta\right)$,

$$
d\left(x_{\varepsilon}, y\right)<r(M)^{2}-2 \varepsilon \delta+\varepsilon^{2}<r(M)^{2} \quad(0<\varepsilon<2 \delta) .
$$

Thus if $\varepsilon<\min (\varepsilon, 2 \delta), \max \left\{d\left(x_{\varepsilon}, y\right), y \in M\right\}<r(M)$. This is contrary to the definition of $r(M)$. Let $\Pi\left(x_{0}\right)$ be the $k$-dimensional affine subspace of $E^{n}$ spanned by $S\left(x_{0}, r(M)\right)$, where $1 \leqslant k<n$. Take $k+1$ points $y_{0}, y_{1}, \ldots, y_{k}$ from $S\left(x_{0}, r(M)\right)$ such that $y_{1}-y_{0}, y_{2}-y_{0}, \ldots, y_{k}-y_{0}$ are linearly independent. Then they obviously span $\Pi\left(x_{0}\right)$. If $k=n$, there is no point except $x_{0}$ whose distances from $y_{0}, y_{1}, \ldots, y_{n}$ are $r(M)$. Hence let $1<k<n$. We will prove $x_{0} \in \Pi\left(x_{0}\right)$. 
Suppose that $x_{0} \notin \Pi\left(x_{0}\right)$. We may assume that $x_{0}=0$ and $\Pi\left(x_{0}\right)=$ $\left\{\left(a, x_{2}, \ldots, x_{k+1}, 0, \ldots, 0\right)\right\}$, where $a$ is a positive constant. Set $I=$ $\left\{\left(x_{1}, \ldots, x_{n}\right), \frac{3}{4} a<x_{1}\right\}$. Then $S\left(x_{0}, r(M)\right) \subset I$ and $M-I$ is compact. Hence we have $\max \left\{d\left(x_{0}, y\right), y \in M-I\right\}=r_{1}<r(M)$. Let $x_{\varepsilon}=(\varepsilon, 0, \ldots, 0) \in R^{n}(0<\varepsilon$ $<a)$. Then we can prove

$$
\max \left\{d\left(x_{\varepsilon}, y\right), y \in M \cap I\right\}<\sqrt{r(M)^{2}-\frac{\varepsilon^{2}}{2}}<r(M) .
$$

Let $0<\varepsilon<\min \left(\left(r(M)-r_{1}\right) / 2, a\right)$. It follows that

$$
\max \left\{d\left(x_{e}, y\right), y \in M-I\right\}<r(M)-\frac{\varepsilon}{2}<r(M) \text {. }
$$

Thus we obtain $\max \left\{d\left(x_{\varepsilon}, y\right), y \in M\right\}<r(M)$. But this is contrary to our assumption. Now, $x_{0} \in \Pi\left(x_{0}\right)$. Then there is only one point on $\Pi\left(x_{0}\right)$ whose distances from $y_{0}, \ldots, y_{k}$ are $r(M)$ as similarly as in the case $k=n$. Lastly we assume that there is a point $x_{1} \notin \Pi\left(x_{0}\right)$ with $B\left(x_{1}, r(M)\right) \supset M$. But this is impossible because it follows from this assumption that at least one of $d\left(x_{1}, y_{i}\right)(i=0,1, \ldots, k)$ should be greater than $r(M)$. The proof is now completed exactly.

3. Proofs of Theorems 1 and 2. Firstly we assume that $M$ and $N$ satisfy the hypotheses of Theorem 2. Moreover suppose that $M$ is isometrically immersed in $N$ and contained in a ball of radius $d$. Then we have $r(M)<d$. Take $x_{0} \in N$ and $y_{0} \in M$ satisfying $r(M)=d\left(x_{0}, y_{0}\right)$. Let $\gamma:[0,1] \rightarrow N$ be a minimal geodesic with $\gamma(0)=x_{0}, \gamma(1)=y_{0}$. For each unit tangent vector $v \in T_{y_{0}} M$, there is a unique Jacobi field $V$ along $\gamma$ such that $V(0)=0, V(1)=v$. Corresponding to $V$, we have a one-parameter family of geodesics from $x_{0}$ to $M, \gamma_{s}(t)=\gamma(s, t):(-\varepsilon, \varepsilon) \times[0,1]$, which satisfies $\gamma_{0}(t)=\gamma(t),(\partial \gamma(s, t) / \partial s)(0, t)=V(t)$. We set

$$
L\left(\gamma_{s}\right)=\frac{1}{2} \int_{0}^{1}\left\langle\gamma_{s}^{\prime}, \gamma_{s}^{\prime}\right\rangle d t
$$

Then from the definition of $\gamma$, it follows that $L\left(\gamma_{s}\right) \leqslant L(\gamma)$. Hence

$$
0 \geqslant\left(\frac{d^{2}}{d s^{2}} L\left(\gamma_{s}\right)\right)_{s=0}=I(V, V)+\left\langle\alpha(v, v), \gamma^{\prime}(1)\right\rangle,
$$

where $\alpha$ is the second fundamental form of $M$ in $N$, and $I($,$) is the index form.$ Taking a proper Jacobi field on a space of constant curvature $b$, J. D. Moore [2] proved that $I(V, V) \geqslant r(M) C(b, r(M))$. Hence from (4) we get $\left\langle\alpha(v, v), \gamma^{\prime}(1)\right\rangle<$ $-r(M) C(b, r(M))$. Since $\left\|\gamma^{\prime}(1)\right\|=r(M)$, we obtain for all unit vectors $v \in T_{y_{0}} M$, $\|\alpha(v, v)\|>C(b, r(M))$. On the other hand, if $\sigma$ is a plane element which is spanned by $v, w$ and satisfies (2), it holds that

$$
\langle\alpha(v, v), \alpha(w, w)\rangle-\|\alpha(v, v)\|^{2}=K(\sigma)-K^{*}(\sigma) \leqslant C^{2}(b, d) .
$$

Since the function $C(b, d)$ is monotonically decreasing, it follows that $C(b, d) \leqslant$ $C(b, r(M))$. Thus the proof of Theorem 1 is finished by the following lemma which was proved essentially by T. Otsuki [4]. We will prove by an argument due to T. A. Springer [2, Chapter 8, §4]. 
LEMMA. Let $\alpha: R^{p} \times R^{p} \rightarrow R^{m}$ be a symmetric bilinear mapping and $\langle,>$ be a positive definite inner product on $R^{m}$. If there is a nonnegative constant $C$ which satisfies

$$
\langle\alpha(v, v), \alpha(w, w)\rangle-\langle\alpha(v, w), \alpha(v, w)\rangle\left\langle C^{2}, \quad\langle\alpha(v, v), \alpha(v, v)\rangle>C^{2},\right.
$$

or

$$
\begin{aligned}
\langle\alpha(v, v), \alpha(w, w)\rangle- & \langle\alpha(v, w), \alpha(v, w)\rangle\left\langle C^{2},\right. \\
& \langle\alpha(v, v), \alpha(v, v)\rangle>C^{2}, \quad \alpha(v, v) \neq 0,
\end{aligned}
$$

for all nonzero $v, w \in R^{p}$, then we have $m \geqslant p$.

Proof. We extend $\alpha$ to a symmetric complex bilinear mapping of $C^{p} \times C^{p} \rightarrow$ $C^{m}$. The equation $\alpha(z, z)=0$ is equivalent to a system of $m$ quadratic equations. If $m<p$, then this system of $m$ equations has a nonzero solution $z=x+\sqrt{-1} y$, where $x, y \in R^{p}, y \neq 0$. As $\alpha(y, y) \neq 0$, from $\alpha(z, z)=0$, we get $\alpha(x, x)=\alpha(y, y)$ $\neq 0$ and $\alpha(x, y)=0$. This is contrary to our assumption.

Theorem 1 follows easily from Theorem 2 . Let $M$ be a compact Riemannian manifold with diameter $d$. If $M$ is isometrically immersed in $N$, it should be contained in some ball of radius $d$. Thus we have Theorem 1 .

\section{REFERENCES}

1. H. Jacobowitz, Isometric embedding of a compact Riemannian manifold into Euclidean space, Proc. Amer. Math. Soc. 40 (1973), 245-246.

2. S. Kobayashi and K. Nomizu, Foundations of differential geometry, Interscience Tracts in Pure and Math., no. 15, Interscience, New York, 1969.

3. J. D. Moore, An application of second variation to submanifold theory, Duke Math. J. 42 (1975), 191-193.

4. T. Otsuki, On the existence of solutions of a system of quadratic equations and its geometrical application, Proc. Japan Acad. 29 (1953), 99-100.

Department of Mathematics, faculty of Education, Tokushima University, Josanjma, TOKUSHIMA 770, JAPAN 